\title{
Minimally invasive resection and reconstruction of the intrathoracic trachea and carina
}

\author{
Gregor J. Kocher, Patrick Dorn \\ Division of General Thoracic Surgery, Bern University Hospital, University of Bern, Bern, Switzerland \\ Correspondence to: Gregor J. Kocher, MD. Division of General Thoracic Surgery, University Hospital Bern, CH - 3010 Bern, Switzerland. \\ Email: gregor.kocher@insel.ch. \\ Provenance: This is an invited Editorial commissioned by Section Editor Jianfei Shen, MD (Department of Cardiothoracic Surgery, Taizhou Hospital \\ of Zhejiang Province, Wenzhou Medical University, Taizhou, China). \\ Comment on: Li J, Wang W, Jiang L, et al. Video-assisted thoracic surgery resection and reconstruction of carina and trachea for malignant or benign \\ disease in 12 patients: three centers' experience in China. Ann Thorac Surg 2016;102:295-303.
}

Submitted Sep 28, 2017. Accepted for publication Sep 29, 2017.

doi: $10.21037 /$ jtd.2017.10.04

View this article at: http://dx.doi.org/10.21037/jtd.2017.10.04

\section{Introduction}

In their publication "Video-assisted thoracic surgery resection and reconstruction of carina and trachea for malignant or benign disease in 12 patients: three centers' experience in China", $\mathrm{Li}$ and co-authors very nicely presented their know-how and techniques of performing these complex procedures by video-assisted thoracoscopic surgery (VATS) (1). They clearly showed the feasibility and safety of VATS in the treatment of benign as well as malignant diseases of the carina and trachea.

The abovementioned study is a good example of the increasing use of VATS approaches also for complex thoracic surgical procedures, which is mainly attributable to the observed reduction in complication and mortality rates after minimally invasive lung resections, particularly among frail patients. In many general thoracic centers, the use of VATS is approaching or has even surpassed open thoracotomy for pulmonary anatomical resections. Motivated by the excellent long-term results after sleeve procedures concerning improved survival, quality of life, reduced loss in lung function, and improved operative mortality compared to more extended resections, the handling of central bronchial, carinal and tracheal operations was improving over time and the experience of performing bronchoplastic resections was growing continuously at the same time.

Until recently, sleeve lobectomies were performed through a thoracotomy. Even in most centers with wide experience in VATS, the surgical indication for a sleeve resection precluded a minimally invasive approach. So far, only a few papers have reported on VATS sleeve resections and have suggested that also these demanding surgical operations may be performed with either conventional multiport VATS $(2,3)$, or even uniportal approaches (4). The report of surgical resection and reconstruction of the central airways (i.e., intrathoracic trachea and/or carina) are even rarer.

\section{Challenges of minimally invasive thoracic surgery in general}

During the last two decades, minimally invasive surgical techniques are increasingly used worldwide in the field of thoracic surgery and other specialties due to important advantages such as lower complication rates, less pain, shorter hospital stay and even a trend versus better survival for early stages of non-small cell lung cancer (NSCLC). This technical step forward in the field of thoracic surgery naturally encourages further development and motivates to reschedule most open procedures as VATS resections (5). Nevertheless, when compared to thoracotomy, VATS procedures turn out to be more technically demanding, mainly because of the transmission from an operating field with multiple angle and especially instrument options and with direct view, into a two-dimensional flat screen with limited access to the operation site. 


\section{Challenges of minimally invasive surgery of the central airways}

Tumors invading the distal trachea or carina additionally represent a challenge due to the complexity of airway reconstruction and management through a thoracoscopic approach. Furthermore, sufficient oxygenation of the patient has to be maintained at any time during surgery. Generally, it seems that, with improvements in VATS competency, greater exchange of knowledge and technical know-how, and advances in equipment, increasing number of centers are able to perform such extended operations by a thoracoscopic approach $(6,7)$. Thanks to a promising evolution of VATS techniques and even of devices, as for example endoscopic knot tying devices or barbed suture technology for bronchial anastomosis, further improvements can be expected in the near future (7-9). The reduction of anastomotic tension and the right choice of the type of reconstruction are the two main concerns when dealing with tracheal and/or carinal reconstruction, regardless of the approach (open or VATS) $(8,10,11)$.

\section{Perioperative ventilation during surgery of the central airways}

As mentioned above, sufficient oxygenation of the patient is one of the main cornerstones, especially when operating on the more central airways such as the carina and/or trachea. To perform central airway resections and reconstructions, there are basically two options in order to maintain lung ventilation: (I) an intra-surgical field tracheal tube or; (II) ventilation by high frequency jet ventilation (HFJV).

In the first option, a sterile circuit is passed through the operative field and prepared to directly ventilate a single lung. Despite of central airway surgery being usually more complex and time-consuming procedures, the additional cross-field ventilation is a successful option in different central tracheoand carinoplasties, and is therefore used in the vast majority $(12,13)$. One of the disadvantages of this technique is, that the cross-field tube can sometimes obstruct the view of the reconstruction site, thus periodical retraction of the tube may sometimes be necessary when performing airway anastomosis in order to improve exposure (3). In case of a VATS approach it is therefore also advisable to insert the cross-field tube through a separate thoracic incision (1). On the other hand, the cuffable tube prevents fluid (i.e., blood) from spilling into the distal airway, which could lead to obstruction of the lung that actually needs to be ventilated.
In case of HFJV, the catheter can be easily introduced through the endotracheal tube and thanks to its small diameter obstruction of the view of the anastomotic site is far less problematic. Furthermore, no additional manipulations including the possible need for an additional incision for introduction of the cross-field ventilation tube are needed (11,14-17). However moderate ventilation of the lung on the operative site may be observed which may affect optimal exposure, especially when using a minimally invasive approach. Additionally, there is a certain risk of barotrauma which in rare cases can result in an acute respiratory distress syndrome (ARDS) $(3,18,19)$.

As He et al. summarized their experience, the extra surgical trauma created by an additional incision for crossfield ventilation was compensated by more stable vital signs, a better exposure and shorter operative time, which in the end resulted in less 'overall trauma' for the patient (3). Regarding the best approach for airway management in carinal and tracheal resections, the last word has not yet been spoken. Additionally, advantages of newer airway techniques have still to become evaluated in a prospective manner. The most important and well-established fact on this issue is, that an optimal collaboration and coordination between the surgeon and the anesthesiologist is essential when performing surgery of the central airways, regardless of the technique and approach that is used $(20,21)$.

\section{Advantages of the minimally invasive approach}

Numerous studies, although mostly retrospective, were able to show a significant benefit of VATS over open surgery concerning acute postoperative as well as chronic pain and/ or numbness at the thoracic incision site (22). Recently also randomized controlled studies reported a reduction in postoperative pain, and significantly better self-reported quality of life during the first year after surgery when a minimally invasive approach was used (23). Another rapidly developing and increasingly used technique is singleport (uniportal) VATS, which holds great promises in further reducing surgical access trauma and postoperative discomfort. The involvement of less intercostal spaces further reduces postoperative chest wall pain, complications and length of hospital stay compared with standard 3-port VATS $(24,25)$.

\section{Closing remarks}

In the end, these technically demanding operations remain 
challenging despite of the operative approach used for it, associated with acceptable but also significant morbidity and mortality risks. Careful preoperative evaluation is the key factor, not only for successful resection and reconstruction, but also for improving postoperative outcomes. Therefore, patients with thoracic malignancy involving the carina or distal trachea should be evaluated as possible surgical candidates based on disease extent and functional status. The minimally invasive approach seems to be a promising option for these resections in the hand of experienced VATS-surgeons, but future studies are needed to further evaluate the safety and especially morbidity rates as well as long-term outcomes after such minimally invasive procedures.

\section{Acknowledgements}

None.

\section{Footnote}

Conflicts of Interest: The authors have no conflicts of interest to declare.

\section{References}

1. Li J, Wang W, Jiang L, et al. Video-assisted thoracic surgery resection and reconstruction of carina and trachea for malignant or benign disease in 12 patients: three centers' experience in China. Ann Thorac Surg 2016;102:295-303.

2. Bertolaccini L, Viti A, Terzi A. Moving beyond the boundary: the emerging role of video-assisted thoracic surgery for bronchoplastic resections. J Thorac Dis 2014;6:1170-2.

3. He J, Wang W, Li J, et al. Video-assisted thoracoscopic surgery tracheal resection and carinal reconstruction for tracheal adenoid cystic carcinoma. J Thorac Dis 2016;8:198-203

4. Gonzalez-Rivas D, Fernandez R, Fieira E, et al. Uniportal video-assisted thoracoscopic bronchial sleeve lobectomy: first report. J Thorac Cardiovasc Surg 2013;145:1676-7.

5. Vannucci F, Gonzalez-Rivas D. Is VATS lobectomy standard of care for operable non-small cell lung cancer? Lung Cancer 2016;100:114-9.

6. Xu X, Chen $\mathrm{H}$, Yin $\mathrm{W}$, et al. Thoracoscopic half carina resection and bronchial sleeve resection for central lung cancer. Surg Innov 2014;21:481-6.
7. Zhao G, Dong C, Yang M, et al. Totally thoracoscopic tracheoplasty for a squamous cell carcinoma of the mediastinal trachea. Ann Thorac Surg 2014;98:1109-11.

8. Nakagawa T, Chiba N, Ueda Y, et al. Clinical experience of sleeve lobectomy with bronchoplasty using a continuous absorbable barbed suture. Gen Thorac Cardiovasc Surg 2015;63:640-3.

9. $\mathrm{Ng}$ CS. Thoracoscopic sleeve resection-the better approach? J Thorac Dis 2014;6:1164-6.

10. Mitchell JD, Mathisen DJ, Wright CD, et al. Clinical experience with carinal resection. J Thorac Cardiovasc Surg 1999;117:39-52; discussion 52-3.

11. Regnard JF, Perrotin C, Giovannetti R, et al. Resection for tumors with carinal involvement: technical aspects, results, and prognostic factors. Ann Thorac Surg 2005;80:1841-6.

12. Maniwa Y. Surgical treatment of air way disease. J Thorac Dis 2016;8:E78-82.

13. Shin S, Park JS, Shim YM, et al. Carinal resection and reconstruction in thoracic malignancies. J Surg Oncol 2014;110:239-44.

14. Gonzalez-Rivas D, Yang Y, Sekhniaidze D, et al. Uniportal video-assisted thoracoscopic bronchoplastic and carinal sleeve procedures. J Thorac Dis 2016;8:S210-S22.

15. Weder W, Inci I. Carinal resection and sleeve pneumonectomy. J Thorac Dis 2016;8:S882-S8.

16. Watanabe $Y$, Murakami S, Iwa $T$, et al. The clinical value of high-frequency jet ventilation in major airway reconstructive surgery. Scand J Thorac Cardiovasc Surg 1988;22:227-33.

17. Nakanishi R, Yamashita T, Muranaka K, et al. Thoracoscopic carinal resection and reconstruction in a patient with mucoepidermoid carcinoma. J Thorac Cardiovasc Surg 2013;145:1134-5.

18. Jaquet $Y$, Monnier P, Van Melle G, et al. Complications of different ventilation strategies in endoscopic laryngeal surgery: a 10-year review. Anesthesiology 2006;104:52-9.

19. Rea F, Marulli G, Schiavon M, et al. Tracheal sleeve pneumonectomy for non small cell lung cancer (NSCLC): short and long-term results in a single institution. Lung Cancer 2008;61:202-8.

20. Hatipoglu Z, Turktan M, Avci A. The anesthesia of trachea and bronchus surgery. J Thorac Dis 2016;8:3442-51.

21. Schieren M, Bohmer A, Dusse F, et al. New Approaches to Airway Management in Tracheal Resections-A Systematic Review and Meta-analysis. J Cardiothorac Vasc Anesth 2017;31:1351-8.

22. Kwon ST, Zhao L, Reddy RM, et al. Evaluation of acute and chronic pain outcomes after robotic, video-assisted 
thoracoscopic surgery, or open anatomic pulmonary resection. J Thorac Cardiovasc Surg 2017;154:652-9.e1.

23. Bendixen M, Jorgensen OD, Kronborg C, et al. Postoperative pain and quality of life after lobectomy via video-assisted thoracoscopic surgery or anterolateral thoracotomy for early stage lung cancer: a randomised controlled trial. Lancet Oncol 2016;17:836-44.

Cite this article as: Kocher GJ, Dorn P. Minimally invasive resection and reconstruction of the intrathoracic trachea and carina. J Thorac Dis 2017;9(11):4319-4322. doi: 10.21037/ jtd.2017.10.04
24. Ng CS, Lau KK, Gonzalez-Rivas D, et al. Evolution in surgical approach and techniques for lung cancer. Thorax 2013;68:681.

25. Harris CG, James RS, Tian DH, et al. Systematic review and meta-analysis of uniportal versus multiportal videoassisted thoracoscopic lobectomy for lung cancer. Ann Cardiothorac Surg 2016;5:76-84. 